\title{
Requirements Gathering for Tools in Support of Storyboarding in User Experience Design
}

\author{
Qiong Peng \\ Eindhoven University of Technology \\ Eindhoven, the Netherlands \\ q.peng@tue.nl
}

\author{
Jean-Bernard Martens \\ Eindhoven University of Technology \\ Eindhoven, the Netherlands \\ J.B.O.S.Martens@tue.nl
}

\begin{abstract}
Storytelling is often used support communication in early User Experience (UX) design, and the resulting stories are frequently visualized in the form of storyboards. However, storyboarding, the creation of storyboards, has also been identified as an important obstacle to the adoption of storytelling in UX design. Most UX designers do indeed not possess the drawing skills needed to produce professionally-looking storyboards. A problem that can be potentially overcome (or at least ameliorated) by using alternative (digital) storyboarding tools. A study into the user acceptance of existing tools is introduced in this paper. The results show that while traditional tools like pen and paper are still very much valued, digital tools are getting increasingly popular. We identify important limitations of existing tools and formulate requirements for future storyboarding tools.
\end{abstract}

Storyboarding, Design Tools, User Experience Design, Storytelling, Communication

\section{INTRODUCTION}

This paper is inspired by observations made in a number of workshops (about 20) conducted with the Storyply [1] method. Some of these workshops involved professional design teams, while others were conducted with teams of design students. These observations support the assumption that storytelling is indeed a valued technique in the conceptual design of user experience (UX), especially if it is supported by a structured method such as Storyply. In order for the method to be effective, however, at least one team member (or the moderator) need to be skilled in storyboarding, as the process of making storyboards is perceived by many participants, especially those that lack drawing skills, as the major bottleneck for adopting the Storyply method. In this paper, we therefore investigate which tools are currently available that could support the creation of storyboards, especially for designers with limited drawing skills. We study the user acceptance of such tools, and identify requirements for future storyboarding tools.

The existing literature on storyboarding tools mainly focuses on tool development, more specifically by taking advantage of new interaction technologies [2-6] such as multi-touch, Augmented Reality (AR) and Virtual Reality (VR). Very little effort is usually dedicated to studying whether or not such tools actually succeed in supporting designers in storyboarding, let alone that they answer our research question as to whether or not such tools could be used in the conceptual design of user experiences.

In this paper, an explorative study into existing tools in support of storyboarding for early UX design is conducted. The purpose is twofold, i.e., to get an overview of tools currently in use as well as to better understand their usefulness for storyboarding in early UX design. Our three main research questions are the following:

Q1: Which tools do UX designers currently use for storyboarding, and what motivates them to use these specific tools? (what and why)

Q2: How do these tools support storyboarding and how well are the outcomes produced by such tools appreciated? (utility, usability and satisfaction)

Q3: What are the limitations of existing tools for storyboarding and how to deal with them? (proposed improvements and future design requirements)

We first discuss in more detail our motivation for conducting this study. Next, we introduce the research methods and outline the process, after which we analyze the findings from our explorative study. Last but not least, we identify design requirements for future storyboarding tools. These requirements can also be used to identify concrete opportunities for improving existing tools. 


\section{BACKGROUND AND REALTED WORK}

It has been observed before that stories are key to understanding user experience (UX). Marc Hassenzahl points out that a user experience is subjective, context-dependent and dynamic over time [7]. He proposes that an experience is actually a story [7-9] that we value sufficiently to share it with others. Glebas in turn believes that stories can convince in ways that logic cannot [10], which is one of the reasons why books, plays and films have a continued attraction on people. In short, a story is a well-established way of sharing and communicating emotional experiences.

A story has a plot that develops from a beginning, where the characters and context are established, through a middle, where conflicts are identified and developed, towards an end where the consequences of proposed actions and interventions are revealed. The proposal of "design as storytelling" [11] has also attracted many designers and researchers. More specifically, Queensbery and Brooks have proposed stories as media in user experience design, as they connect product ideas to the people who will use the product [12]. Gruen et al. have similarly advocated the use of stories in user experience design [13]. McCarthy and Wright highlight that telling stories is a way of making sense of experiences [14]. Others regard storytelling as an effective way to gain a holistic view on the targeted user experience [15]. Storytelling can help to deliver rich information as it provides details about the characters and the contexts in which products or services are supposed to demonstrate their value. Crafting stories has therefore been proposed as a core competency when describing and communicating UX [16].

UX design is the design of a product, system or service to enhance positive appreciation [17] such as user satisfaction and/or pleasure. It forces designers to focus on the emotional impact of design ideas. Early UX design is a creative process which alternates between idea generation and problem solving. Communication of and reflection on ideas are the main activities in early UX design. Frequent methods for visualizing and communicating design ideas is through sketches, prototypes and/or storyboards. Sketches are easy and fast, but tend to focus on (static) product features, and moreover require skills of hand drawing that many people do not possess. Prototyping requires a substantial effort and time investment by technically-skilled personnel, and may moreover not be warranted at early conceptual stages. Storyboarding employs low-tech means to not only describe ideas for products, systems and services but to also make the context of use and the emotional impact on the users explicit. Storyboards not only visualize design ideas but help to create a common understanding about the problem to be solved and the targeted experience of the people involved.

Storyboarding is defined here as the creation of a storyboard that is a visual depiction of a narrative [18] or a story by a series of sketches, photographs, animations or pictures [19], possibly accompanied by text, speech or sounds. Storyboarding has a long tradition in the production of movies, TV, cartoons and animations [20]. Although storyboarding has often been promoted within the area of UX design, there is a lack of research on actual practice. Most of the literature stresses the usefulness of sketching and camera shot techniques [10, 20-24], but fails to validate that such techniques indeed match the skills and interests of existing UX designers. Next to the technical aspect of making storyboards, experts and novices alike also face additional problems when trying to apply storyboarding in UX design. It is indeed not an easy task to create a storyboard within a couple of minutes that not only presents a product idea (what it is) but also clarifies its impact (why it is relevant). This might be the underlying reason for the problem mentioned before that storyboarding is considered to be the main bottleneck for adopting storytelling in UX design. Making use of tools that can support storyboarding is therefore a potential solution. Appropriate tools do not only have the potential to assist in the creation of storyboards but may also help to produce a more professionally-looking output that is more likely to be shared with stakeholders.

Existing storyboarding tools can be divided into two main categories: (1) the still much-used traditional tools like pen and paper, and (2) digital tools that involve specialized software, on-line platforms and/or mobile apps. The digital tools can in turn be subdivided into professional and non-professional. The former tools, such as Storyboard Pro, Storyboarder and ToonBoomStoryboard Pro, are frequently used by professional storyboard artists in the production of films and animations. We will however focus on non-professional tools, including tools for storyboard sketching or animation, picture and video editing, etc., as they are less expensive, more general purpose and are a priori expected to be more likely candidates for adoption by designers (as will be confirmed by our first study).

A review paper on storyboarding tools by Salim et al. [25] describes and analyses 24 storyboarding tools, concepts and frameworks from the perspective of teaching storyboarding in e-learning environments. The paper does however not go into the actual use of storyboarding tools, let alone from the specific perspective of UX design. The paper by Truong et al. [18] highlights important characteristics of storyboards as guidelines for successful storyboarding. It does however not pay 
any attention to the role of tools. Some recent publications focus on how to create storyboards by means of new digital technologies, such as augmented reality (AR) [5]. The AR Storyboard tool [3] is for instance helpful in scene composition and camera motion control. It therefore seems fair to conclude that the existing research approaches offer only a limited perspective as they focus on the making of storyboards, largely ignoring the actual use and effectiveness of storyboards, especially for presenting and discussing UX concepts.

There is no definition of storyboarding tools specifically for UX design. Any tool that supports storyboarding by either sketching or by pictures, and even by video or animation, are usually viewed as storyboarding tools. There is hence a variety of tools that could support storyboarding in very different ways. In this study, we make an exploration of existing tools in an attempt to understand whether or not such tools facilitate storyboarding in an adequate way. The insights collected from this explorative study will subsequently be used to identify directions for improving existing tools and for developing alternative new ones. They will also provide a more detailed understanding of why storyboarding is perceived as a bottleneck for adopting storytelling in UX design.

\section{METHODOLOGY}

We conducted two separate studies. Firstly, in order to get to know how designers view existing tools for storyboarding, an online questionnaire was distributed, and answers by UX designers and design students were collected. The questionnaire contained both closed and open questions, the latter ones addressing aspects such as: which tool(s) do you use and for what purpose. We also asked the participants to recommend one favourite tool and to explain their arguments in favor of their choice.

The tools mentioned in the first study were categorized and ranked, and a selection of representative tools was selected to be included in a follow-up study. The selection included both sketchbased and image-based techniques, as well as combinations of the two. This second study included both an online storyboarding contest and a workshop with students from our design department. Computers and iPads with the tools already installed were provided to the participants in the workshop, while the online users were provided with internet links with download instructions.

Participants in both the online contest and the locally conducted workshop were required to produce a storyboard based on a given UX design task. At the start, they were provided with a story script and had to choose one of the recommended tools. The difference between both conditions was that the participants in the online contest worked individually and separately (with unlimited time) while the participants in the workshop worked as a team in the same room on the same storyboarding task. A USE questionnaire (Usefulness, Satisfaction and Ease of use) [26] was filled in by all participants. In the USE questionnaire, statements had to be scored on a seven-point Likert scale ranging from 1 "Strongly disagree" to 7 "Strongly agree". An interview was conducted with the workshop participants after finishing the storyboarding task. In this interview, questions were asked about subjective opinions on the tool, suggestions for improvements, as well as advice for alternative new tools.

\section{RESTULTS}

\subsection{Results of study 1}

The online questionnaire was filled in by 60 participants mainly from the Netherlands (47\%), China $(40 \%)$, the USA $(10 \%)$ and the others $(3 \%)$. The participants were either UX designers $(58 \%)$ or students (42\%) being trained in UX design. All of them had experience in storyboarding as a way to support communication in early UX design. We distinguished their storyboarding experience into expert $(70 \%)$, in case they had more than one year of storyboarding experience, or novice $(30 \%)$, in case the storyboarding experience was less than one year. Sketching only was preferred by $10 \%$ of the participants, while $15 \%$ indicated to use only pictures; $75 \%$ of the participants used a combination of both approaches.

The participants in the questionnaire proposed a range of different storyboarding tools. As figure 1 shows, traditional pen and paper was mentioned by all participants, while the Adobe software programs Illustrator and Photoshop are in use by $40 \%$ and $30 \%$ of the participants respectively. PowerPoint and Keynote were also liked by the same percentage $(30 \%)$ of people, as were web-based platforms (such as StoryboardThat, Pixton) and mobile apps (such as Procreate and Paper 53). The professional tool Storyboard Pro was cited by only $5 \%$ of the participants.

The reasons provided for using specific tools for storyboarding in early UX design were also analyzed. UX design practitioners adopt tools like PowerPoint/Keynote, StoryboardThat, Photoshop etc. for presentations, describing and explaining design ideas to stakeholders. Tools like Procreate, Paper 53 and pen\&paper were mainly used for exchanging and discussing ideas internally. The different tools were ranked in terms of frequency of occurrence and the top ten is listed in Figure 2, together with some of the reasons for why they were recommended. 


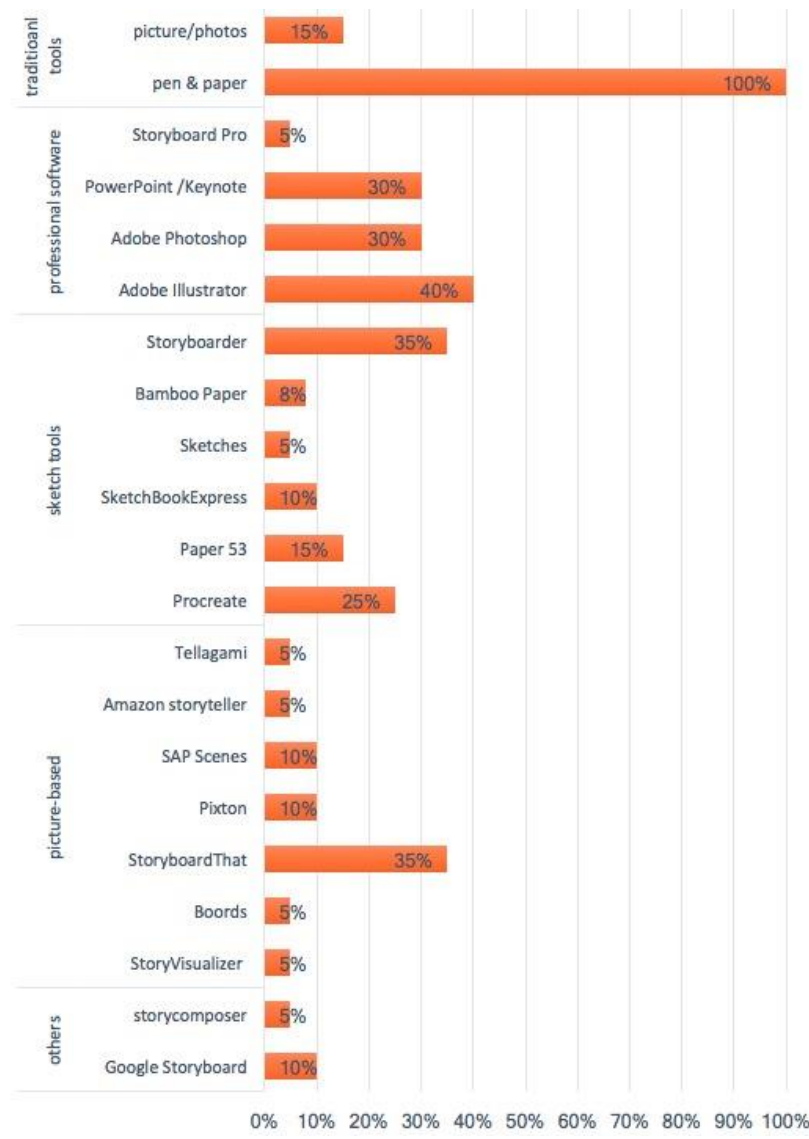

Figure 1: The tools mentioned in the online questionnaire and the percentage of participants who mentioned the tool

A selection of the above tools needed to be made for a follow-up, more in-depth, study. The generalpurpose tool of pen-and-paper, the two Adobe software programs (Illustrator and Photoshop), and the presentation software PowerPoint, were all excluded in our selection, partly because we were interested in digital tools which contained specific storyboarding functionality.

Two picture-based tools were selected: StoryboardThat, a web-based tool for storyboarding with pictures, and Pixton, a storyboarding tool with pictures, available as both a web-based and an iPad app. Two sketch-based tools were selected: Procreate, an iPad sketch app and 2013 Apple design award winner, and SketchBookExpress, an iPad sketch app. These 4 recommended tools were included in study 2.

\subsection{Results of study 2}

15 students participated in the storyboarding consisted of a mixture of undergraduate students, master students and PhD students studying industrial design, and used to UX design projects. They rated their experience with storyboarding on a seven-point Likert scale, ranging from 1 (totally unfamiliar) to 7 (totally familiar). As mentioned before, students with more than 1 year experience in using storyboards were classified as experts $(76.2 \%)$ while students with less than 1 year of experience were classified as novices (23.8\%).

The selection of the 4 tools was as follows: StoryboardThat (42.9\%), SketchBookExpress (28.6\%), Pixton (19\%) and Procreate (9.5\%). A factor analysis revealed that gender, age and level of experience of the participants did not have a significant effect on their tool choices.

\begin{tabular}{|c|c|c|c|}
\hline Rank & Tools & Percentage & Examples of the reasons \\
\hline 1 & $\begin{array}{l}\text { Pen } \\
\text { paper }\end{array}$ & $25 \%$ & $\begin{array}{l}\text { "I mostly make storyboards by hand sketching with pen and paper." } \\
\text { "Pen and paper are available for sketching for storyboards at any time." } \\
\text { "Easy to use and convenient" }\end{array}$ \\
\hline 2 & Illustrator & $20 \%$ & "Mllustrator provides vector drawing tools to sketching flexibly." \\
\hline 3 & Photoshop & $18.3 \%$ & "Photoshop has powerful functionalities for picture edition". \\
\hline 4 & Procreate & $15 \%$ & $\begin{array}{l}\text { "2013 Apple design award winner" } \\
\text { "easy-of-use on iPad" }\end{array}$ \\
\hline 5 & Storyboard-That & $8.3 \%$ & $\begin{array}{l}\text { "providing a lot of images stored in its library" } \\
\text { "StoryboardThat is helpful for me because I am not good sketching." }\end{array}$ \\
\hline 6 & PowerPoint & $5 \%$ & $\begin{array}{l}\text { "easy to insert, delete, place and edit images for storyboarding" } \\
\text { "easy and available animation effects" }\end{array}$ \\
\hline 7 & SketchBookExpress & $3.3 \%$ & "features in sketching like layer, and brush choices" \\
\hline 8 & Pixton & $1.7 \%$ & "Pixton helps me for storyboarding without drawing. It's interesting to use this tool." \\
\hline 9 & Paper 53 & $1.7 \%$ & I used Paper 53 for storyboarding for many years and never changed for others \\
\hline 10 & Storyboarder & $1.7 \%$ & "Storyboarder has different functions and much useful and helpful for my work." \\
\hline
\end{tabular}

Figure 2: The top-10 tools with their recommendation percentages and some reasons for recommendation 
The winning storyboards from the online contest in both categories (picture-based and sketch-based) are shown in figure 3 and figure 4.

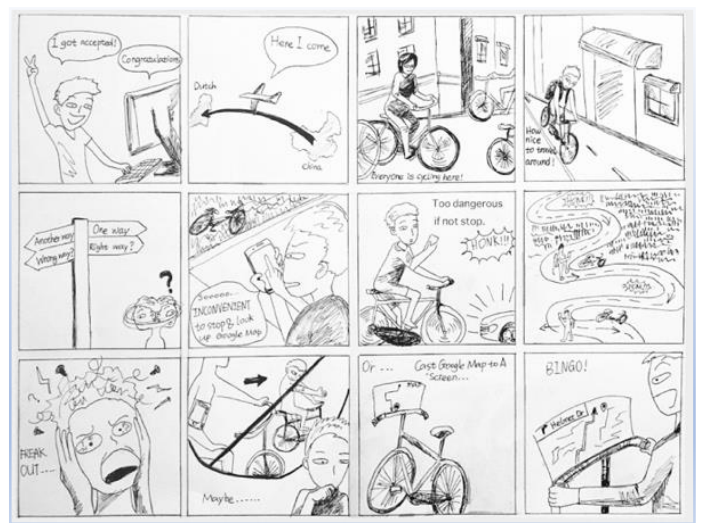

Figure 3: The winning storyboard (sketch-based)
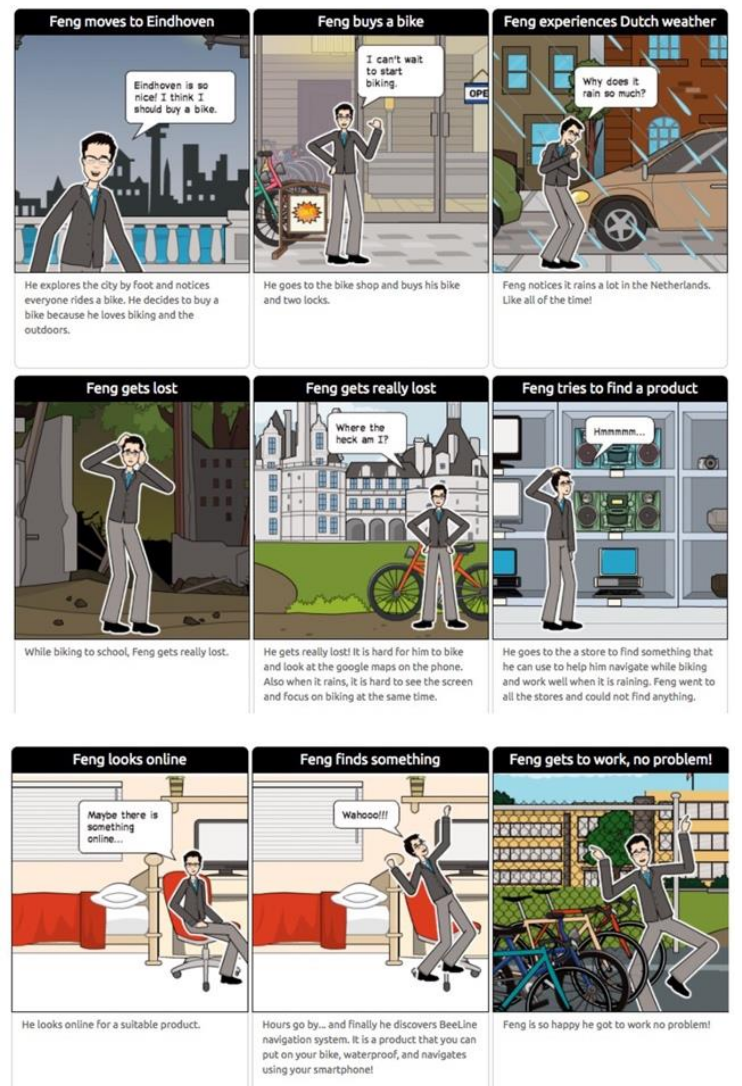

Figure 4: The winning storyboard (picture-based)

The results of the USE questionnaire were analyzed with a new program for interactive statistics called ILLMO [27]. A difference between this program and more traditional statistical programs such as SPSS, is that ILLMO is very visually oriented, which makes it easier to learn for users who are relatively new to statistics. The most relevant feature of the ILLMO program for the current discussion is that it contains some advanced clustering algorithms.

The 30 questions in the questionnaire were first analyzed individually in order to establish whether or not an individual question contains significant differences between at least two of the four tools being rated (Tool 1: StoryboardThat, Tool 2: Pixton, Tool 3: Procreate and Tool 4: SketchbookExpress). Questions that did not contain such significant differences between any pair of tools were obviously uninformative and were removed from the analysis. Only 11 of the 30 questions in the original questionnaire were retained in this preprocessing stage. A $1 D$ clustering of these 11 questions was subsequently performed in order to find clusters of questions that behaved in a similar way. This resulted in 3 clusters:

Cluster 1 is labelled as "Meet Expectation" and contains 6 of the original questions (Q17: It did everything I expected it to do, Q22: It was flexible, Q35: It was fun to use, Q37: It was wonderful, Q38: I feel I need to have it (I would use it again) and Q39: It was pleasant to use). The internal consistency between the questions in this cluster is excellent, as expressed through a Cronbach's alpha of 0.956 (and an average pairwise correlation of 0.791 ). As shown in the left graph of Figure 5, Tool 3 receives the highest average score for the questions in this cluster. Cluster 2 is labelled as "Useful" and contains 4 of the original questions (Q11: It helped me to be more productive, Q15: It saved me time when I used it, Q25: I didn't notice any inconsistencies as I was using it (sub-activities were performed in a similar/consistent way) and Q29: I learned to use it quickly). The internal consistency between the questions in this cluster is also excellent, as expressed through a Cronbach's alpha of 0.946 (and an average pairwise correlation of 0.814 ). Tool 2 receives the highest score for the questions in this cluster. Cluster C3 contains only a single question, Q15: It was simple to use, as the corresponding pattern. It is significantly different from the answers on any of the other questions.

In order to understand the relationship between the different questions and clusters, 2D clustering was also performed. The result for the three 1D cluster patterns and for the original 11 questions are displayed in Figures 5 and Figure 6, respectively. The length of the vectors in these figures are proportional to the squared correlation between the average cluster/question score and the projection onto the displayed 2D plane, where the dotted circle indicates perfect correlation.

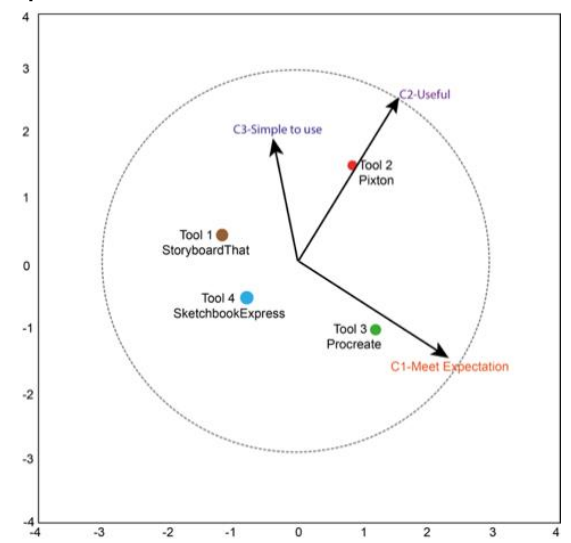


Figure 5: $1 D$ cluster derived from the retained 11 questions from the questionnaire, presented as vectors in a $2 D$ plane.

In Figure 5, the clusters C1and C2 have correlations that are almost equal to 1 , implying that the $2 \mathrm{D}$ graphical representation adequately summarizes the average cluster scores. As the correlation for cluster C3 is substantially smaller than 1, the 2D graphical representation is however only an approximation of these average cluster scores. Geometrically, this means that the vector representing cluster C3 is not within the plane but points either towards or away from the viewer.

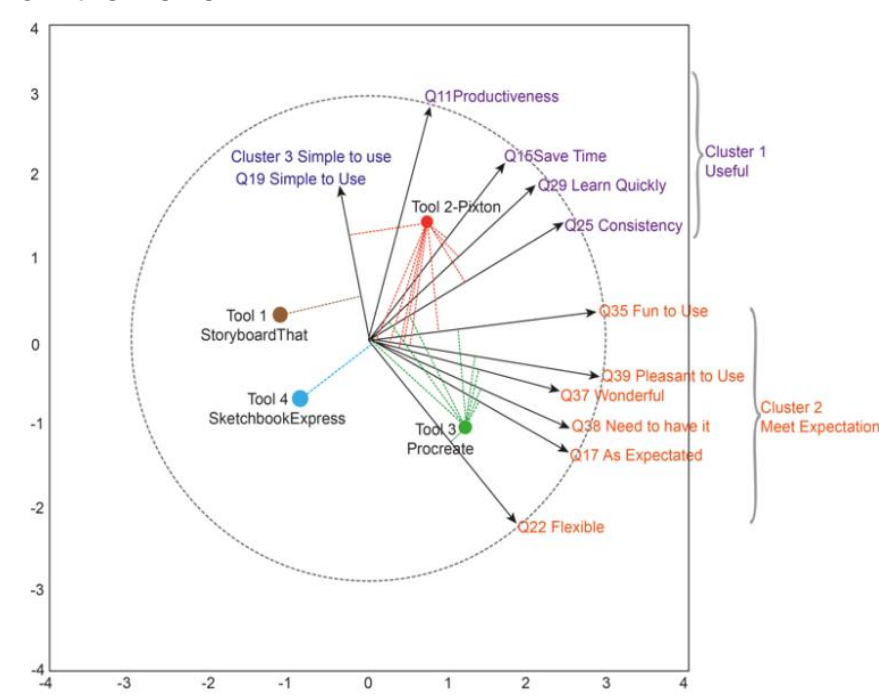

Figure 6: Individual items from the questionnaire used in the internal storyboard contest are represented as vectors in a $2 D$ plane. The vectors representing items in different clusters are represented by distinct colours.

We can produce an alternative 2D clustering, shown in Figure 6, where the 11 retained items from the questionnaire are rendered individually. All 11 items are rendered simultaneously and the lengths of the black vectors are proportional to the squared correlation between the average scores on the different items and the predictions as derived from the 2D graph (i.e., these predictions are the orthogonal projections of the tools, represented by the points, onto the vectors representing the questionnaire items). From figure 8 we can deduce that Tool 2 (Pixton) scores highest for items in cluster 2 (which mostly contains items that have a relationship to usefulness) and Tool 3 (Procreate) score highest for items in cluster 1 (which mostly contains items that confirm that the tool meets the expectations).

From the picture-based tools, Tool 1 (StoryboardThat) and Tool 2 (Pixton), the latter tool is clearly the preferred one (although it is not the one that is selected the most). From the sketch-based tools, Tool 3 (Procreate) and Tool 4 (Sketchbook Express), the former tool is the preferred one (again, it is not the one that is selected the most). Tools 2 and 3 are therefore the most likely candidates to start from when trying to develop improved tools. Tool 2 (Pixton) could be improved by increasing the score on items in cluster $\mathrm{C} 1$ (meets expectations), while Tool 3 (Procreate) could be improved by increasing the score on items in clusters C2 (useful). Note that Tool 2 is also simpler to use than Tool 3.

\subsection{Results of the interviews}

The answers in the interviews were firstly transcribed verbatim and individual quotes were extracted and labelled. To guarantee the consistency of the coding process, 3 researchers, including the first author and 2 other researchers who are familiar with qualitative content analysis but not with the specific aims of the study, were involved in the coding process.

We identified 27 quotes that were related to the need for new storyboarding tools; $88.8 \%$ of them expressed supportive attitudes to alternative tools. For instance, one participant believed that "It is necessary to have some better new tools" while another one stated "It is urgent to develop new tools which can better support storyboarding". The remaining $11.1 \%$ quotes contained only obscure attitudes such as "I am not really sure" or "I have no idea".

We identified 198 quotes related to strengths and weaknesses of the selected tool; 85 quotes addressed positive aspects while 113 quotes provided more details about weaknesses. The analysis of these negative judgements revealed four categories:

(1) Device limitation (6 quotes), for instance, "it is limited to be used only on iPad", or "only a web app is so bad, because I mostly work with PC";

(2) Functionality (43 quotes), examples are "I cannot edit my sketch", "fewer Undo steps" and "too limited brushes for sketching";

(3) Interface design (17 quotes), examples are "ugly interface design", "the layout of the tools is too bad to find and use";

(4) Communication with (19 quotes), for instance, quotes such as "I cannot export my sketches in different format like PDF or JPG picture" and "it does not provide immediate communication and file-sharing with others".

The finding of the tools' deficiencies indicated participants' dissatisfaction about these tools and also highlighted the possibility for improvement.

The 96 quotes with concrete suggestions for improvements were divided into 5 categories: Functionality (37 quotes), Open-Resource (25 quotes), Compatibility (14 quotes), Help-Support (13 quotes), and Customization (7 quotes). These five categories were further analyzed into sub-categories to provide an overview of suggestions for improvements and desired features in future tools. 
The most prevalent quotes in the different categories are the following:

(1) Functionality: One quote said that "It should provide more tools for sketching". Another one proposed "to combine the functionalities in both StoryboardThat and Procreate";

(2) Open-Resource: Accessibility of external sources is important, for instance, one quote proposed "to upload and download the templates freely", another stated that "It should provide online searching for necessary images";

(3) Compatibility: The focus was on different platforms and media. The quotes highlighted aspects such as "to use the tool both on mobile devices and PC" and that "audio and visual resources to be used together";

(4) About Help-Support: The quotes emphasized many different aspects such as "provide instruction especially for new beginners", "sometimes need to ask my friends for help when storyboarding", "sharing through different methods like email, social media", and "storyboarding with team members together";

(5) Customization: The quotes addressed meeting different individual needs, for instance "the tool should provide different choices for different kind of users" and "it should provide the functionality for personal setting like setting the favourite sketching brushes".

\section{DISCUSSION}

The results from study 2 indicate that none of the recommended tools performed optimally on all relevant attributes. Hence, it is worthwhile discussing design directions for future storyboarding tools, either by improving existing tools or by design alternative tools.

Before entering into details, we should be aware that storyboarding is an example of a creative process, and that tools for storyboarding should hence possess features that have been identified as being important for tools supporting creativity [28], such as supporting exploration, low threshold for both novices and experts, fun to use, etc. As designing storyboarding tools is in itself an example of UX design, different contexts of use and distinct user characteristics, such as novice vs expert, individual vs team, sketch-based vs picture-based, co-located vs remote cooperation, etc., will also influence the prioritization of wanted functionalities.

\subsection{Design direction 1: Classification and optimization of functionalities}

Tools for storyboarding can be sketch-based, picture-based and a combination of the two. No matter which style is adopted, it is important to comply with features that people have gotten used to from general-purpose programs such as Photoshop, Illustrator, PowerPoint, etc. Such features can be classified into different categories: drawing (brushes, colours, lines, eraser), image editing (move, add, delete, rotate, insert, resize, zoom in/out), image import/export, communication (chat, inviting, messages), collaboration (sharing, cosketching, upload, download), organization (preview, overview, image files) and others like searching. They can be optimized per category, for instance, adding missing ones (like Undo, filter, layer) to image editing, or providing more brushes and colours to support drawing or making it more convenient for sharing by offering more options rather than only email.

Another option for optimization of functionalities is from the perspective of specific attributes. Based on figure 6, we can conclude that Tool 2 (Pixton) and Tool 3 (Procreate) are superior to the other two (Tool 1: StoryboardThat and Tool 4: SketchbookExpress). It therefore makes sense to consider optimization starting from these two tools that users already like. We should however keep in mind that there is also one aspect, simple to use in cluster $\mathrm{C} 3$, for which inspiration can be drawn from another tool (Tool 1). In Figure 7, the two areas (purple and orange) indicate the major design dimensions. Based on Tool 3 (Procreate) which scores best in terms of the items in cluster C1 (meet expectation), it could probably be improved on aspects such as efficiency (i.e., aspects such as saving time which are part of cluster $\mathrm{C} 2$ in the purple area).

Since Tool 3, Procreate, is a sketch-based tool, users think it is flexible and fun to use. However, if users are not skilled in sketching, the possibility to include pictures (or sketches that are automatically derived by processing images) may be a valuable way to improve the visual quality of storyboards and/or may save substantial time. Hence, improvement in functionalities that are inspired by picture-based tools, such as easy input, output and editing of pictures, could be added to make Procreate more productive and suitable for the task of creating storyboards.

The feedback from participants indicates that Tool 2 (Pixton) provides efficient means for constructing storyboards, but produces an output that looks rather childish, which makes it less suited for professional applications, especially when considering presentations. The items in cluster $\mathrm{C} 1$ indicate that sketches seem to be much more acceptable. Providing opportunities for sketching on top of storyboards produced by Pixton, up to the point where the original storyboards are completely hidden, may be a way to successfully combine positive features of both tools. 


\subsection{Design direction 2: Hybrid storyboarding support and cross-device interaction}

There is no single perfect tool that can meet all user needs. However, the four recommended tools each have their own attractive properties. The strengths and weaknesses of tools in both the sketch-based and picture-based categories are compared in table 1.

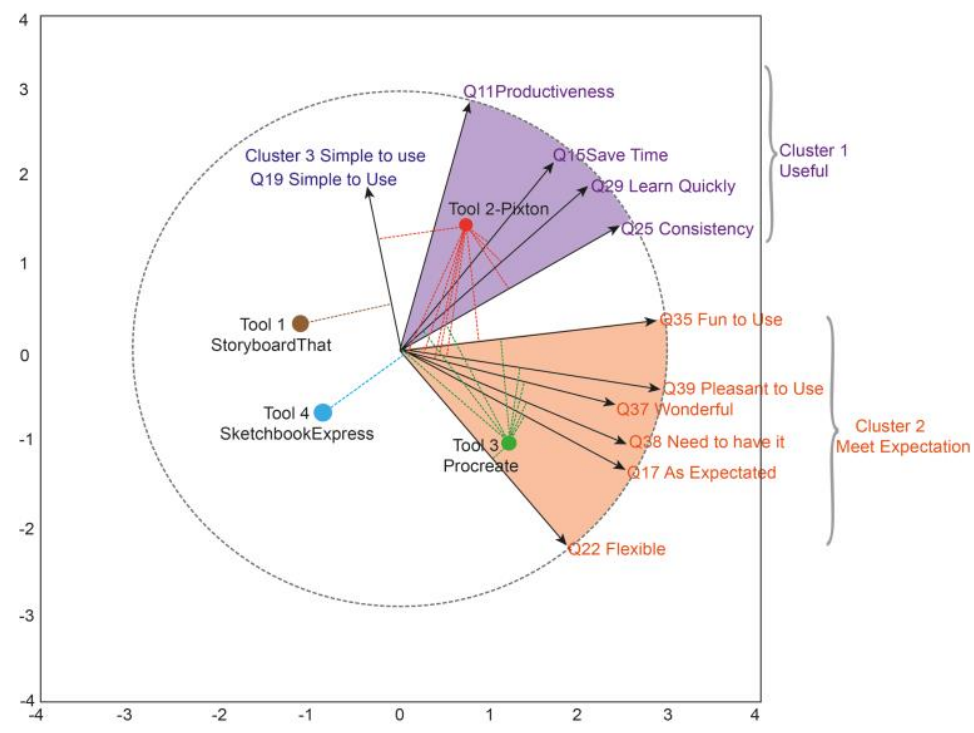

Figure 7: Design space and direction of the storyboarding tools provided by the four tools

Table 1: Comparison of the sketch-based tools and picture-based tools in terms of merits and defects

\begin{tabular}{|l|l|l|}
\hline Category & Merits & Defects \\
\hline \multirow{3}{*}{$\begin{array}{l}\text { Sketch- } \\
\text { based }\end{array}$} & freedom & Need drawing skills \\
\cline { 2 - 3 } & Self-control & Output quality \\
\cline { 2 - 3 } & rapid & Personal style \\
\cline { 2 - 3 } & inspiring & Not easy to understand \\
\hline \multirow{3}{*}{$\begin{array}{l}\text { Picture- } \\
\text { based }\end{array}$} & good quality & time-consuming \\
\cline { 2 - 3 } & Easy to use & $\begin{array}{l}\text { limitation in supporting } \\
\text { detials }\end{array}$ \\
\cline { 2 - 3 } & No sketch skill & style-consistence \\
\cline { 2 - 3 } & $\begin{array}{l}\text { Free picture } \\
\text { choices }\end{array}$ & learn to use it first \\
\hline
\end{tabular}

Picture-based tools are useful and productive because they are convenient, especially for users who are not skilled in drawing, as they use readily available pictures as building blocks. As sketchbased tools require drawing skills, they are mostly appreciated by users who favor the freedom of expression in storyboarding. According to the feedback received in the storyboard contest, $63 \%$ of the participants believed that the optimal storyboarding solution should combine sketches and pictures. In practice, many people use multiple tools for storyboarding. Hence, it makes sense to discuss hybrid tools that use pictures but that also support sketching. Users should be offered the freedom to use either or both of these two approaches. For example, if users don't know how to draw by hand, they should be provided with the option to take photos, download or import pictures as templates and make modifications to such material rather than having to start from a blank canvas. This is sometimes called hybrid sketching [21], as it combines sketched elements with photos Another example is where users can make storyboards with pictures or icons stored in the tools and then add more details such as dialogues, emotions and other necessary elements by means of sketching.

At this point, it is also important to point out that storyboards are not necessarily restricted to sketches and pictures. In user experience design, including animations[28] and videos may be a viable alternative. Hence, there are also opportunities for new hybrid storyboarding tools that take full advantage of not only sketches and pictures, but also alternative media such as speech music, animations and videos.

Hybrid storyboarding can also be supported by improving the compatibility with existing tools. For instance, allowing designers to edit images in professional software like Photoshop for better visual effect and export them to tools which are more suitable for creating storyboards may be a way to combine the best of both words. It would also avoid the painful (and error-prone) process of copying (and debugging) well-established methods into a new storyboarding program.

Another feedback that we received is worthwhile to highlight in this discussion. The feedback implied that the tools could have more impact provided that they were supported on multiple platforms or devices. People are increasingly getting used to across-device interactions that combine traditional 
media such as pen on paper with digital devices such as mobile phones, tablets, PCs, big multitouch screens, electronic whiteboards, and increasingly also augmented reality (e.g., based on Kinect), as provide new means of creating and presenting content. It is also in accordance with recent literature stating that digital technology is an "excellent means of promotion and distribution" [5]. Such flexible combinations of diverse technologies within prototypes (sometimes called "hashing" in the field of design) are also ideally suited to support storyboarding in the context of UX design. Considering that the primary purpose of using storyboarding in UX is for communication (discussion and presentation of design ideas), the efficiency and effectiveness of content sharing and annotation should definitely be taken into account when judging the design of new tools.

\subsection{Design direction 3: Open-resource supporting collaborative storyboarding}

An insight gained both from the observations and the interviews in the storyboarding workshop is that it is not unusual to seek external support for storyboarding, as UX design nowadays is increasingly a team effort. The third design direction for storyboarding tools is hence looking towards crowdsourcing support (either online or within the local community) for collaborative storyboarding. This would mean that part of the project budget would be set aside to finance crowdsourcing competitions that can help in realizing the storyboards that are needed for the project. This means shifting emphasis from creating storyboards to specifying storyboards (e.g., through story scripts).

Members of a design team can potentially also play different roles in storyboard co-construction and modification, and may profit from alternative tools, provided of course that the storyboards can be easily transferred between such tools. Even in the context of co-located team work, open-resource tools can hence be beneficial for collaboration. StoryCrate [6] is a recent example of a system that supports collaboration on a storyboard (for film production) by creating a shared representation on a table-top with a tangible interface.

\subsection{Limitations of the study}

The sample sizes in the studies were relatively small, so that the results are mostly useful for identifying interesting directions for improvement rather than for validation. In study 2, all the participants were limited to being design students from our Industrial Design department (hence, no professional UX designers were involved in this study). The focus of the studies was also on creating storyboards, so that other important aspects, such as communication and collaboration, have not yet been studied in depth.

We noticed that there was quite some overlap between the clustering obtained from the quantitative analysis and the coding and identification of categories in the qualitative analysis, which argues for a more integrated analysis of both types of data, such as using the categories identified in the qualitative analysis to identify a priori clusters of the items in the quantitative analysis, hence implying a priori boundary conditions on the quantitative clustering.

Another limitation was that our studies mainly focused on usability, usefulness and users' subjective opinions on the four recommended tools. Observations of how people actually use their tools during the storyboarding process were obviously not available in case of the storyboarding contest. Some important aspects may hence not be reported as people may not remember or judge as relevant some of the issues that occurred during the process when responding to the questions in the questionnaire or interview.

Key to UX is that an incomplete picture is bound to arise if we only study the usefulness and usability of tools and ignore the use context and user characteristics, such as the cognitive and behavioral abilities of the participants involved in a storyboarding process. For instance, in our study, an obvious confounding factor is that participants who are good at sketching mostly prefer sketch-based tools for storyboarding while participants who are not proficient in sketching prefer picture-based tools. The experience with existing tools and the expectations of the environment are other obvious factors that influence the preference for one tool or the other.

\section{CONCLUSION}

This paper provides several distinct contributions. Firstly, it provides an overview of existing tools that are often used by UX designers for storyboarding. Secondly, it identified design directions for future tools. As part of the research process, we showed how techniques such as cluster analyses of both qualitative and quantitative data can be used to help structure the large amount of gathered experimental data. Moreover, it is shown how a graphical model of the design space, such as in Figures 5, 6 and 7, can be used to identify directions in which to improve existing tools.

In order to solve the problem of storyboarding as a bottleneck for adopting storytelling in early user experience design, the importance of adequate and appropriate tools for storyboarding should not be ignored, which is why we were interested in identifying design directions for storyboarding tools. 
There seem to be three main directions. The first one is to focus on the functionalities of the tools, and to draw inspiration from tools that are already widely adopted. The second direction is to develop hybrid storyboarding tools which can combine the advantages of both sketch-based (such as Procreate) and picture-based (such as Pixton) tools and that support an easy transfer between digital and physical media. The third direction is to involve crowdsourcing into the storyboarding process, shifting the emphasis from making to specifying storyboards and using competitions to optimize results.

\section{REFERENCES}

[1] B. Atasoy and J.-B. Martens. (2016) STORYPLY: designing for user experiences using storycraft, in Collaboration in Creative Design, Springer, pp. 181-210.

[2] R. Singh and A. Solar-Lezama. (2012) SPT: storyboard programming tool, in Computer Aided Verification, 2012, pp. 738-743.

[3] M. Shin, B. Kim, and J. Park. (2005) AR storyboard: an augmented reality based interactive storyboard authoring tool, in Mixed and Augmented Reality, 2005. Proceedings. Fourth IEEE and ACM International Symposium on, 2005, pp. 198-199.

[4] A. Jhala, C. Rawls, S. Munilla, and R. M. Young.(2008) Longboard: A Sketch Based Intelligent Storyboarding Tool for Creating Machinima., in FLAIRS Conference, 2008, pp. 386-390.

[5] C. Pallant and S. Price. (2015) "Storyboarding in the Digital Age," in Storyboarding, Springer, 2015, pp. 151-172.

[6] T. Bartindale, A. Sheikh, N. Taylor, P. Wright, and P. Olivier. (2012) StoryCrate: tabletop storyboarding for live film production, in Proceedings of the SIGCHI Conference on Human Factors in Computing Systems, 2012, pp. 169-178. [7] M. Hassenzahl. (2010) Experience Design: Technology for All the Right Reasons, Synth. Lect. Hum.-Centered Inform., vol. 3, no. 1, pp. 1-95.

[8] M. Hassenzahl. (2013) User experience and experience design, Encycl. Hum.-Comput. Interact.

[9] M. Hassenzahl and N. Tractinsky. (2006) User experience-a research agenda, Behav. Inf. Technol., vol. 25, no. 2, pp. 91-97, 2006.

[10] F. Glebas. (2012) Directing the story: professional storytelling and storyboarding techniques for live action and animation. Taylor \& Francis.

[11] P. Parrish. (2006) Design as storytelling, TechTrends, vol. 50, no. 4, pp. 72-82.

[12] W. Quesenbery and K. Brooks. (2010) Storytelling for user experience: Crafting stories for better design. Rosenfeld Media.
[13] D. Gruen, T. Rauch, S. Redpath, and S. Ruettinger. (2002) The use of stories in user experience design, Int. J. Hum.-Comput. Interact., vol. 14, no. 3-4, pp. 503-534.

[14] J. McCarthy and P. Wright. (2004) Technology as experience," interactions, vol. 11, no. 5 , pp. $42-43$.

[15] H. Korhonen, J. Arrasvuori, and K. Väänänen-Vainio-Mattila. (2010) Analysing user experience of personal mobile products through contextual factors, in Proceedings of the 9th International Conference on Mobile and Ubiquitous Multimedia, 2010, p. 11.

[16] Q. Peng. (2017), Storytelling Tools in Support of User Experience Design, in Proceedings of the $2017 \mathrm{CHI}$ Conference Extended Abstracts on Human Factors in Computing Systems, 2017, pp. 316-319.

[17] I. McClelland. (2005) User experience'design a new form of design practice takes shape, in CHI'05 Extended Abstracts on Human Factors in Computing Systems, 2005, pp. 1096-1097.

[18] K. N. Truong, G. R. Hayes, and G. D. Abowd. (2006) Storyboarding: an empirical determination of best practices and effective guidelines, in Proceedings of the 6th conference on Designing Interactive systems, 2006, pp. 12-21.

[19] R. Sova and D. H. Sova. (2006) Storyboards: a dynamic storytelling tool, in Proceedings of the 2006 UPA conference on Usability through Storytelling, 2006.

[20] J. Hart. (2013) The Art of the Storyboard: A filmmaker's introduction. Taylor \& Francis.

[21] S. Greenberg, S. Carpendale, N. Marquardt, and B. Buxton. (2010) Sketching user experiences: The workbook. Elsevier.

[22] J. A. Landay and B. A. Myers. (1996) Sketching storyboards to illustrate interface behaviors, in Conference Companion on Human Factors in Computing Systems, 1996, pp. 193-194. [23] J. Van Sijll. (2005) Cinematic storytelling: The 100 most powerful film conventions every filmmaker must know. Michael Wiese Productions Studio City, CA.

[24] S. D. Katz. (1991) Film Directing Shot by Shot: Visualizing from Concept to Screen. Gulf Professional Publishing.

[25] S. S. Salim. (2014) A review of storyboard tools, concepts and frameworks, in International Conference on Learning and Collaboration Technologies, 2014, pp. 73-82.

[26] A. M. Lund. (2001) Measuring usability with the use questionnaire12, Usability Interface, vol. 8, no. 2, pp. 3-6.

[27] J.-B. Martens. (2014) Interactive Statistics with IIImo, ACM Trans Interact Intell Syst, vol. 4, no. 1 , p. 4:1-4:28.

[28] J. Quevedo Fernandez. (2017) Sketching animations in design and beyond, Eindhoven University of Technology. 